\title{
An exploration of occupational choices in adolescence: A constructivist grounded theory study.
}

\section{Abstract}

Background: Adolescence is a critical period within the life-course, for developing adult occupational competencies and health behaviours. Few studies have considered how 16 to 17 year olds choose activities and behaviours from an occupational perspective.

Aim and objectives: To explore how adolescents aged 16 to 17 years old make choices about their daily occupations to inform a theoretical model of occupational choice.

Materials and Methods: 27 secondary school students aged 16 to 17 years attended one of six focus groups. Transcripts were analysed using constructivist grounded theory, informing the iterative development of a theoretical model of occupational choice.

Results: Adolescent occupational choice occurred in response to experiencing needs, and was characterized by "weighing up" and "juggling" the following four key domains: 'Appraising values and priorities', 'Interacting with the situational context', 'Exploring skills and occupational repertoire', and 'Considering time factors'. A developing sense of responsibility and autonomy for occupational choices was described, leading to the development of the future occupational self.

Conclusions and significance: A theory illuminating how adolescents make choices was developed. The theory aligns with existing developmental literature and provides unique insights, from an occupational science perspective, on the conscious process by which adolescents make, develop and adapt choices about the occupations they do considering contextual and individual opportunities and constraints. 
Keywords: Activity, Late Adolescence, Occupation, Occupational Balance, Occupational Choice, Time-use, Teenagers, Wellbeing 


\section{Introduction}

Adolescence is widely regarded as a sensitive and critical period of development [1]. The period is associated with neurological, psychological, physiological, emotional, social and economic development [1-3], as well as development of the occupational competence required for adult roles [4]. Today's adolescents are developing in a rapidly changing world facing increased uncertainties such as increased occupational choice, changes to normative working patterns, increased 'free-time', and existing in a culture of individualism as well as a digital online age in which behavioural norms are still being established [5-6]. Health behaviours are established during this critical period, consequently the study of adolescence is of particular interest to those hoping to improve the health and wellbeing of this population [7].

The human experience of health, wellbeing and identity has been linked to the activities or occupations in which people engage [8]. The relationship between what a person does and the experience of health and wellbeing is complex, and is thought to be influenced by the meaning, purpose, value and balance of occupations [9-12]. This occupational perspective is an area of increasing interest to those seeking to promote health and wellbeing. New terms such as 'lifestyle psychiatry' are being used to describe evidence such as the link between engaging in physical activities and mental health [13].

According to a moral and ethical argument, complex interventions designed to improve health and health related behaviours should be based on evidence based logic models [14]. Consequently, effective promotion of healthy occupational patterns and behaviours in adolescence that endure into adulthood require an understanding of the adolescence 
occupational nature, experience and perspectives. The presence of the adolescence perspective is limited, in both the occupational science theory and the empirical research literature. Time-use studies have begun to illuminate the complexity of adolescent occupational patterns in relation to health, but research to date has been mainly quantitative in nature. Furthermore, existing research fails to consider trade-offs between necessary and desired occupations and focuses mainly on single types of activities, for example, the impact of time spent in sedentary activities versus time being physically active [15].

Similarly, the extent to which the empirical base of occupation-focused models reflect the occupational nature of children and adolescence is questioned [16]. This raises the question to what extent adolescent occupational choices are the same or different to that of adults. The concept of 'Occupational choice', defined in the Model of Human Occupation (MOHO) as "deliberate commitments to enter an occupational role, acquire a new habit, or undertake a personal project" $[\mathrm{p} 14,4]$, is also currently debated, particularly by those interested in adolescent or mental health perspectives. 'Occupational choice' as represented by the Model of Human Occupation (MOHO), and also other models such as the Canadian Model of Occupational Performance and Engagement (CMOP-E) or the Person-EnvironmentOccupation-Performance Model (PEOP), is regarded as basically individualistic, with limited conceptualisation of how choices are shaped by the externally situated environmental determinants [17-18]. Taken together, to inform a logic model, we propose the need for research that delves thoroughly into a variety of external factors, and specific examination of the subjective process by which adolescent populations make choices between one activity and another on a daily basis, particularly in late adolescence. 
Late adolescence, typically categorised as aged 15 to 19 years of age [19], is an important educational transition point and a key time for the onset of mental health problems, which typically occur between 14 and 18 years of age [20]. Rising levels of reported mental health problems among adolescents has placed increasing strain on services with few young people receiving timely and effective treatments [21-22]. Untreated or inappropriately treated illness can cause disruption to the normal development of occupational patterns, behaviours and choices with long-term implications for future functioning [23]. Improving our understanding of how young people make choices can inform the development of occupation-based interventions to improve health outcomes during this period.

Consequently, this study focuses on deepening understanding of occupational choice during the critical period at a key educational transition point within many school systems system. The ultimate purpose of the study is to inform the logic model of a larger study to develop an occupational therapy informed, evidenced-based intervention, to improve the mental health of 16 to 17 year olds in a mainstream school environment.

\section{The study aims}

This study aimed to explore the processes involved in choosing occupations from the perspective of late adolescents in mainstream school and to develop a proposed theory to explain these processes.

\section{Materials and method}

A qualitative methodology was chosen for this study, because it is considered to be best suited to understand the experience, meaning and perspectives of participants [24].

Consequently, this methodology enables exploration of how adolescents make choices and 
why. An open-ended, inductive constructivist grounded theory method was selected for its potential to build both substantive and general theories from qualitative data [25-27].

The constructivist approach to grounded theory, proposed by Charmaz, considers knowledge and truth as created rather than objectively knowable, and emerging theory as co-constructed between researcher and participants, through the interactive research process [26-29]. This methodological approach was considered appropriate because it enables exploration of connections, identification of process, construction of theory, and acknowledges the researcher's involvement and impact on the research process. This enables the researcher to conceptualise how young people make occupational choices, and achieve the study aim.

\section{The researchers' characteristics, reflexivity and trustworthiness}

The constructivist perspective acknowledges that studying a phenomenon influences both the data collected and its interpretation, and no consensus view between researchers is considered necessary [29]. The primary researcher (first author), who is from a white British, middleclass background with 19 years clinical experience as an occupational therapist aimed to critically examine personal responses, avoid assumptions not grounded in the data, and ensure fidelity to constructivist grounded theory methods. Three female co-authors provided a critical and analytical reflexive space for the primary researcher to discuss emerging ideas. The background of the co-authors includes; experience of conducting research, a professional qualification as an occupational therapist or physiotherapist, and different cultural backgrounds including British, Swedish and American. 


\section{Study design}

A focus group method was used as it provides an insight into the complexity of everyday interactions and normative assumptions [30], whilst also being considered a less intense and more acceptable method for adolescence than one to one interviews [31]. The method was chosen as it provides an opportunity to engage with the complexity of group dynamics and how people position themselves in their environment [32]. Time constraints related to limited access to the school environment was a further rationale for the use of focus group rather than individual interviews, allowing involvement of more students. A first group was run to pilot procedures and later included in the main study.

\section{Recruitment, sampling \& study procedures}

The first five participants were recruited purposively through conveniently available personal networks for the pilot group. The remaining participants were recruited from two schools involved in multi-phase study to develop an occupational therapy intervention, the first phase of which involves a quantitative study examining time-use in relation to wellbeing. In this earlier quantitative study gaining access to schools for research purposes had been difficult. The primary researcher emailed over 20 local schools inviting them to participate in the study.

The first school recruited was situated in a low income, highly urbanized area with a broad range of ethnic diversity and was the only one to respond to the email recruitment method. A minimum of two schools were need for the earlier quantitative study; therefore a second school was recruited via a teaching consultant who, using her connections, approached further schools about the study. The second school recruited was situated in an affluent semirural location with a mainly white British population. Information was provided to the headmasters of both schools prior to acquiring signed consent for school participation. Gate 
keeping responsibilities were delegated to the heads of year following a research protocol briefing.

Across both schools a total of 135 typical year 12 students (aged 16 to 17 years) participated in the quantitative study and were invited to indicate their interest in being contacted about participating in focus groups. The original research design intended to construct a stratified sampling frame based on adolescent time use and wellbeing from which to purposively select participants. Twenty-three indicated an interest in participating in a focus group. The low opt in rates meant that the planned stratified sampling frame, was not possible. Therefore, all 23 students were invited to participate and given further information before being asked for signed consent. One student indicated an interest in participating but chose not to take part for personal reasons after reviewing the study information and discussing alternative ways to engage. Following the low response rate the pilot group was also incorporated into the analysis and viewed as an earlier iterative stage of the analysis process. A total of six focus groups were conducted in English with a total of 27 adolescents which included the 22 of the 23 who volunteered from the schools and the five participants from the initial pilot group.

\section{The sample description and demographics}

All 27 participants spoke English, had recently completed their General Certificates of Secondary Education (GCSE), attended a mainstream local secondary comprehensive school and were either due to start or had just started the first semester of year 12. Sixteen girls and eleven boys took part, 12 participants came from the semi-rural school and 15 from the urban school, while the pilot consisted of five English speaking, UK born girls. The urban school sample was ethnically diverse with eight participants born outside of the UK (Romania, Russia, India, United Arab Emirates and Afghanistan), and nine of the participants (33\% of the total sample) identified as non-white, such as Black or Asian. Two disclosed that they had 
previously been schooled in India. All participants had previously completed their English GCSE qualification, demonstrated a good understanding of the English language and engaged actively and fluently in English throughout discussions.

\section{Ethical considerations}

The study was approved by a University Research Ethics Committee (UREC no. 181192) and compliant with the good clinical practice (GCP) and requirements of the Helsinki declaration [33]. Those aged 16 and over are considered able to give informed consent to participate in research studies of this nature. However, in the school setting it is considered appropriate to keep parents informed about what occurs within the school environment as those under the age of 18 are still considered to be children. Consequently, parents were also informed about the study.

\section{Data collection}

All participants were briefed on the ground rules, their rights and what to do if they felt distressed while participating. The focus groups were conducted on school premises during self-directed study periods and each participant was given an online shopping voucher for 20 pounds to acknowledge their contribution.

An adapted version of an interactive card sorting activity developed for 13 to 24 years olds called the Activity Card Sort - Adolescent, Children and Young Adult (ACS-AYA) [34] was used as an warm up exercise for the focus groups leading into the semi-structured topic guide (see Figure 1). The tool uses photographs depicting different activities to elicit lived experience of engaging and participating in activities [34,35]. The purpose of using ACSAYA was to promote rapport [36], active participation, engagement in the topic of enquiry 
[37], and develop of rich data. The semi-structured topic guide then formed the main structure of the interviews (see Figure 1) Additional prompt questions were used to explore responses related to the impact of occupational choices. Adaptions were also made to the topic guide between focus groups.

Figure 1 in about here.

There were between two and seven participants in each group, and each audio-recorded session took between 26 and 53 minutes. Audio recordings were transcribed verbatim by the primary researcher as advised by Charmaz [29]. A reflexive diary was kept by the primary researcher throughout the analysis. A second researcher observed and made notes, which the primary researcher used as an aide-memoire for her own reflexive analysis. In order to maintain confidentiality and data security, pseudonyms were used, and all data was kept securely according to the university data protection policies.

\section{Data analysis and saturation}

The analysis was undertaken according to the constructivist grounded theory approach proposed by Charmaz [29]. The pilot group was analysed first, which involved each of the first 4 steps illustrated in Figure 2. Initial theory modelling was explored and was later used in comparative analysis.

Figure 2 in about here.

Due to school timetables and the planned curriculum, access to the schools for the purpose of conducting the main focus groups was limited to one day for both schools and occurred a week apart. Ideally, data is collected and analysed concurrently to enable categories and concepts to be identified, explored and tested through theoretical sampling until the data is saturated and a satisfactory theory developed [29]. The fact that three focus groups were 
completed in one day in the first school, and two in one day in the other school a week later, limited theoretical sampling based on detailed analysis between the data collections, potentially impacting on the iterative process of data generation that is core to grounded theory. To mitigate the limited opportunities for theoretical sampling caused by restricted school access, several actions were taken. Firstly, the topic guide was adapted between the pilot, the groups and between schools, based on initial reflections rather than after detailed analysis of each focus group. Secondly, each focus group was individually analysed from step 1 to step 4 as illustrated in Figure 2 before moving on to the next focus group, enabling questions and ideas to develop which could be explored in the next group. A chart was used retrospectively to record all themes in the order that they emerged during the analysis, which confirmed that no new ideas emerged from the analysis of the last group, thus suggesting that saturation had been achieved.

Once all six transcripts were individually analysed to step 4, a detailed process of constant comparison was undertaken as illustrated in steps 6 and 7 in Figure 2. Focused codes and provisional categories were collated into a spreadsheet that recorded which code emerged from which focus group. A total of 151 individual codes were initially identified. Similarities and differences were compared before being reduced and refined to a core version, for which descriptive definitions were further developed. Specific attention was given to contradictory cases.

Memos and reflexive notes were kept by the primary researcher at all stages of the data collection and analysis process, ensuring attention was also given to the relationships between codes, focused codes and categories. Through constant comparison and moving back 
and forth through the data and memos, four categories appeared to be strongly interconnected and of importance to the adolescent process of making an occupational choice. These main categories would be described as domains and were central to the proposed model. The process of making a choice, appeared to begin with identifying the need and the urge to fulfil it. These emerging ideas were explored further in the data and through various diagrammatic models in an iterative process to develop a cohesive proposed theory, which is described below. General grounded theory principles were followed, which advocate minimal literature reviewing prior to data collection [27]. Instead, literature was reviewed after data collection to develop theoretical sensitivity and originality in relation to existing knowledge.

\section{Results: Developing the future occupational self through occupational choice and occupational experience}

The findings that emerged from the focus group transcripts are presented as a proposed theory. Pseudonyms are used in place of participants' names, quotes are written in italics and 'FG' refers to originating focus group. An overview of the four inter-connected domains that make up the core of the proposed theory is explained first, followed by an explanation of the two sub-processes involved in occupational choice.

The proposed theory starts as the adolescent first experiences a need to engage in occupation for a specific purpose, such as need to connect with others, need to achieve, need to become competent, need to relax and need to develop. This prompts them to engage in a complex 
process of weighing up and juggling at least one of four interconnected domains; Appraising

Values and Priorities, Interacting with the Situational context, Exploring Skills and Occupational Repertoire, and Considering Time factors (see Figure 3 for illustration of the core model of the theory).

Figure 3 about here

The four categories describe four different but interrelated domains that affect the process of choosing what to do. Appraising Values and Priorities refers to the values and priorities that influence the adolescents' choices. Values are made up of beliefs, preferences, opinions, ideas and norms that collectively are reflected in the importance and meaning placed on any given occupation or need. Regarding values, one participant said, "I mean like my parents have done a good job of teaching me good values" Komal (FG2). Priorities, in contrast, refer to the adolescents' prioritization of one occupation or need against another, often based on the value of the occupation or need such as, "Well the things you know you should do you have to do eventually, but you sort of put them off to the last minute. Till you really have to do it" Ben (FG4). The adolescents express that both values and priorities are influenced and based on the socio-culture, gender, and family norms in which the young person lives and develops. As illustrated by Abrial's (FG4) response to a question about how culture shape choices of time use, "It depends...cause my English family are like do what you want but my Dad's family (from Asia) are very how's your school work... and did you get good grades...all my cousins are like sometimes we pull all-nighters and I'm like yeah, I get to 9 and that is enough work for me.” They also appear to reflect the way occupation is classified, judged and prioritized within the community in which the adolescents live, providing standards of competency and mastery by which occupation is perceived to be given a value, participation 
appraised, and experience is judged. For example, one participant said: "I think there is a feeling like when you are relaxing... you have had a really busy day... you are sure you should not be doing anything but you feel you should revise or whatever" Maria (FG5).

The Interacting with the Situational Context refers to the present physical and social situation or environment in which the adolescents finds themselves, and how this affects them. This can also include; specific preferred environments; whom they are with or where they are at a given time; and the resources available to them such as money, internet or the nature of the support provided by others. For example, one adolescent who was sent to the UK by his parents under the conditional that he must perform well in his studies, demonstrates awareness of the impact of his situational context on his choices of occupation: "I personally don't get much pressure at home because I am already aware of what is at stake” Barry (FG3).

Exploring Skills and Occupational Repertoire domain describes the personal resources and skills an individual has, including both their perception of confidence in using their skills and their past experiences of using these skills to meet arising needs. Skills describes the unique abilities required to complete an occupation while the occupational repertoire is the library of experience from which an individual can draw as in this example of learning to cook: "Cooking is a really good activity to do... you learn new things which helps out later in life" Tom (FG5).

Finally, Considering Time Factors domain refers to the multiple aspects of time, such as its perceived value, the amount of time available, deadlines, length of timelines and how well 
the participants feel they manage time. For example: "I think I have... poor time management skills... I will have forgotten about certain homework and then I've realized I have to do it and it might be late into the night that I might start doing something” Barry (FG3).

Weighing up and juggling these domains is then followed by making an occupational choice (sub process 1) and then the experience of engaging in the chosen occupation. The nature of the experience is then appraised/evaluated as to how the experience met the need, leading the adolescent to weigh up and juggle the experience in relation to the four domains. This results in nuanced and personalized version of the domain: "If someone is trying to tell me to do something I personally don’t listen. I think I know myself. I know what I am doing is the correct way of doing things" Gemma (FG3). These domain modifications have the potential to influence future occupational choices and contribute to the construction of the emerging occupational self (sub-process 2).

\section{Detailed Model Description}

The model presented in Figure 4 illustrates the two model sub-processes; Making a choice and doing occupation, and the Emerging occupational self, which are added to the core model presented earlier in Figure 3. The two separate sub-processes are the active processes involved in choosing an occupation, and are connected through the four central domains already described.

Figure 4 about here

\section{Sub-Process 1: Making a choice and doing occupation}

This process involves the adolescent 'weighing up' and 'juggling' the four domains, with the purpose of informing which occupation to choose to best meet the identified need. 


\section{Domain: Appraising values and priorities when making a choice and doing}

occupation

The adolescent engages in a process of weighing up the value and priority status of the identified occupational need and any potential occupations that may meet that need.

Simultaneously they also engage in a process of juggling the external values and priorities that structured and guided earlier stages of development, and their own internalised and emerging values and priorities, as illustrated by Gurdip (FG3): "If you think it's wrong, you think twice like should I do it, should I not do it and study?" Values and priorities appear changeable and can be influenced by the other three domains, such as the availability of time, the adolescents' skill level and the context in which the choice and occupation take place.

Adolescents appear more likely to ascribe a higher priority status to occupations in which they find enjoyment. As the participant Charles (FG4) articulates, "if you like something more than something else, you are obviously going to do the thing you like more obviously than the thing they should do." There is a clear sense of their ability to articulate and provide a rationale for their own priorities, occupational preferences, value statements, and judgements about what occupations they choose, including how they meet specific needs or purposes. Almost universally, occupations were discussed in terms of the adolescents' experience of doing the occupation, but adolescents described and grouped occupations into what are widely-recognized western occupational classifications such as work or leisure. Occupations were frequently judged in relation to their potential to provide or enable future work roles, as illustrated by Alan's (FG3) statement, "Work is priority, because work is the future." 


\section{Domain: Interacting with the situational context when making a choice and doing occupation.}

Adolescents juggle multiple and sometimes conflicting aspects of the situational context that surrounds them which can be experienced as influencing, supporting, obstructing or enhancing in relation to both choosing occupation and meeting one's needs. Emma (FG5) expressed the following regarding her drama class: "Well I've stopped now, but when we did shows and stuff for years which was fun... it was just fun to like meet new people. And like put on shows and that sort of thing....they changed the teacher and I didn't like the new teacher." At the same time, they experienced juggling the ongoing transition from externalized control to personal autonomy and responsibility.

The participants identify the impact of multiple environmental aspects including culture, religion, gender, upbringing, social norms, romantic relationships, peers, family, family history and expectations. The nature of the support they receive from others, whether informal or formal, impacts their occupational choices. Appropriate support at the right time can encourage experimentation with new occupations, which may or may not meet their needs, while the inadequate support from others may discourage some occupations. How support is perceived and experienced can impact the likelihood of an occupation being maintained over time or stopped: "When they ask me if I have anything to do or if they nag me then I don't do it, just to annoy them." Jess (FG5).

The situational context can also be enhanced or obstructed affecting the process of choosing an occupation. Various obstructions exist, for example parental rules about where and when a young person can go out, or limited availability of public transport. Situational context can also refer to experimenting with personally preferred environments, such as where an 
occupation is best preformed. Examples include noise levels, type of surroundings, and preferred company: “During my exams I went to the library every day. It didn't work... It was more distracting for me. I study better when I am at home.” Gurdip (FG1). Situational context also includes the resources available such as financial resources and external demands, or requirements like as meeting homework deadlines. For example Liz (FG2) used to play the violin but is no longer able to because of her situational context, "I used to play violin and I don't do it here... we don't have a guide... who can guide us...my Dad loves it because his family is all into music... and I am the only one now that's not doing it so he gets upset I don't do it anymore...it's really expensive to get someone"

Limited autonomy and independence necessitates that the adolescents weigh up the best response to their situational context and any restrictions. Responses include accepting, agreeing to, conflicting with, adapting by choosing another occupation, rebelling, deceiving, or concealing a preferred occupation behind a more acceptable occupation. They may also engage in bargaining or negotiating around occupational decisions, such as what Natalie (FG1) describes: "Sometimes I have to negotiate with them and then they will eventually let me, like the other day or the next day."

\section{Domain: Exploring skills and occupational repertoire in making a choice and doing occupation}

In making a choice the adolescent must weigh up what physical, cognitive, and emotional abilities they have as well as the skills they do or do not yet have to complete an occupation. Occupational skills are many and varied including the ability to act, play football, sustain relationships, wash oneself or care for a pet. Skills can also include self-knowledge such as knowing personal limits, how to assess risk, and knowing which occupation to use to meet an 
arising need, as well as judging competencies and capabilities. Adolescents engage in weighing up their confidence in their own skills, and the likelihood that current skills will successfully meet their arising need(s). For example, Anna (FG0) states:

I want to start this new job. I need to think about what I'm going to be, where my head is going to be at in 6 months' time and whether I am going to be able to cope with playing rugby, going to work, and helping out at boys brigade...like doing all my Alevels and things...it's the first thing you think of and it's kind of frustrating having to let go of things.

The adolescent draws on historical experiences of making choices about occupations and their subsequent experience of doing that occupation; a personalized narrative is constructed of past experiences of how needs were met by chosen and unchosen occupations. This is illustrated by Simon (FG1):

Yeah [going to the gym] is my favourite activity. I used to hate exercising but since I started gym, I feel more healthy and stuff so... I go five days a week... Yeah kinda makes a difference I feel more healthy. It changed my diet as well... I didn't feel that good before gym. I was like let me try gym for the first time... it wasn't so good after the first two weeks. I felt the change after the first two weeks or three weeks so.

Past successes and failures have shaped perceptions of skills, competencies, and selfknowledge. Consequently, perceptions of past occupational experiences, choices and selfknowledge are weighed up when making an occupational choice. This is evidenced by a comment by Gurdip (FG1): "Everything that we do throughout our lives we've done it before, even whether you like it or not you've done it before." By this he was illustrating a relationship between how current skills build on past experiences of doing. 
Collectively, the adolescent's unique set of occupational skills and abilities, history of occupational experiences, knowledge and perception of their capabilities forms their repertoire of occupations. Adolescents appear focused on developing their repertoire of occupations, have expectations of their capability to do so and provide examples of developing new skills, which affects their choice of occupation. The perceived distance between the adolescent's current skill level and the level required to successfully complete an occupation appears important when making these choices. Occupations may be avoided if the adolescent perceives them to be too challenging because they do not have the required skills, are uninteresting or consider the occupation boring because they are already competent in the skills required for the occupation. For example, one boy described an occupation he used to do with his mother to develop his writing skills: "Creative writing... I thought it was good but it's one of those things that I could live without" Tom (FG5). This suggests that there may be an optimum exposure and level of challenge for each individual with a given occupation that is more likely to result in a positive experience and continued engagement in that occupation.

The skills and occupational repertoire must again be juggled alongside one or more of the other three domains when making a choice of occupation. For example, values and priorities may affect how much effort an adolescent is prepared to put into learning a new skill. In addition, time factors may affect perceived competence to complete an occupation, and context may affect the perceived challenge of an occupation.

\section{Domain: Considering time factors when making a choice and doing}

The final aspect of the making a choice is the time domain, which includes weighing up and juggling the time available, the time an occupation takes, balancing multiple demands on 
one's time, and organizing occupational priorities. As illustrated by what David (FG4) explains about giving up cricket. "It takes too long... [I stopped] last summer when I could be doing other stuff during the day...it likes takes too much time... well it felt like you're doing a lot of nothing when you could be doing more productive things." Time has been given a value and is considered an important asset requiring careful management to avoid waste. Adolescents experience a sense of increased autonomy over their time-use as they grow older. For example, Mathew (FG4) talks about his studies in relation to time-related stress, and refers to "the preciousness of time." He continues, "but it's not really necessarily that important right now but later on we will be like we need more time". The participants reported needing to weigh up meeting immediate and longer-term needs, and how the short term may affect long-term needs; for example, homework is linked to exam performance, and ultimately career choices. A complex juggling process occurs between differing needs, and levels of urgency, which affects how the individual engages with an occupational and how they feel, as described by Jess (FG5):

It's quite hard to relax when you have school for 6 hours a day and then you go home and you do homework. And I dance from 6 to $9: 30 \ldots$ And then... come home... have a shower and go to bed. ...I have my work... on a Saturday... and then I go out... and I don't have time to do anything else.

The adolescent constantly has to weigh up their use of time and the level of balance achieved between different occupations at any given time. They have increased freedom to use their time wisely or unwisely as this adolescent describes:

I've got in to a bad habit of not getting enough sleep... so I'll work late... sometimes it's due to how long the work is... I'll get 5 or 6 hours of sleep and then... I'm getting myself pumped on caffeine and then coming into school... it hurts my head sometimes. Gemma (FG3). 


\section{Domain: Weighing up and juggling the interconnected nature of the four domains.}

When choosing an occupation the adolescent appears to weigh and juggle multiple factors within each domain, and also the interconnected nature of the four domains, when making a choice. For example the situational context of an occupation may change, thus affecting the occupation's priority and value, which, when weighed against time constraints, may result in an occupation being deliberately dropped or rejected, impacting on skill development. As described by one adolescent who explained why she gave up playing the piano: "I played the piano for about 10 years. I just stopped because the piano teacher wasn't very nice. So now I've basically quit altogether... It's not that I don't like it...it's just that I don't have time for it anymore." Jess (FG5). This in turn may lead to conflict with family members or others who are unhappy with the adolescent's choice.

Other examples of the interconnected nature of the domains include deciding to learn a new skill, which may be affected by availability of time, situational context, skills and occupational repertoire domains. Values and priorities may be affected by the situational context, such as when out with friends or at home with family. The past experience of doing occupations influences how a person views or values those occupations, resulting in avoidance of occupations associated with negative performance experiences, despite the adolescent's potential capability to complete the task. The experience may have been strongly affected by the situational context, such as the level of support from others. How the adolescent weighs up and juggles these domains affects the experience of how well their needs are met through a chosen occupation, potentially impacting on feelings of wellbeing. This is illustrated by Kimali (FG3) who, despite finding that sport involvement helps her 
anxiety, explains the challenge she experiences in getting both sleep and finding time to play sport:

If I do my homework I don't get enough sleep, because there is too much homework... I can't survive the day without 7 hours sleep so if I do my homework... I do not get time to sleep... when I come back from school, I go on the TV, I watch something, I eat something and after that I go have a bath and freshen up...then I go to start studying but in this thing like I don't get to play sports...you don't get enough time to yourself.

Adolescents' choices may have a detrimental impact, such as when choosing to leave school work to the last minute may result in the individual experiencing pressure, avoiding occupations and generally failing to meet personal needs. "I don't recognise that I feel like I am constantly kind of under pressure but then I recognise it when it gets too much. So it's like too late before we recognise it if that makes sense." Jess (FG5). If they manage to juggle domains effectively, they appear more likely to experience a sense of balance with in their occupational experience.

\section{Sub-Process 2: The Emerging Occupational Self}

The second sub-process concerns the process of appraising the experience of engaging in the chosen occupation, selected through process 1 , and shapes the transition towards a more selfgoverned occupational self. The occupational self can be described as emerging, because the four domains which were previously structured or influenced by a "scaffold" created by significant people such as family, teachers or friends. This structure was initially experienced as both compulsory and necessary to establish patterns and routines of occupation, but during adolescence is gradually replaced by a more personalized version of the four domains in readiness for adulthood. Specifically, the adolescent appears to adopt many aspects of the external scaffold but the reliance on the scaffold appears to decrease. This occurs as they take a greater level of ownership for making their own occupational choice and do so increasingly 
autonomously. For Example Nora (FG0) talks about her reading behaviours: "But when you get older, I just think as soon as I started secondary school, I stopped reading, except if I was on holiday." This transition is further outlined in relation to each of the four domains.

\section{Domain: Appraising values and priorities in the development of the emerging self}

The experience of engaging in a chosen occupation offers the adolescent the opportunity to weigh up how the occupation met their values and priorities, and to juggle between the values and priorities of the world around them (the external scaffold) on which they have previously shaped their views and their own emerging ideas and values. Adolescents may choose to align more firmly with external values or reject them. For example, one participant talked about wanting to play the drums but ultimately giving up the occupation: "I used to play drums to be specific and it's been a long time since I stopped it" and he highlighted the impact of culture and his father's opinion on that decision: "it depends on the culture... like Asian family is like more strict and stuff." Simon (FG1). They demonstrate an awareness of increased autonomy to make their own choices about what occupations they do and their right to accept or reject externally provided values and priorities. "When I was on year 9 and 10 they would ask me if I have any homework and stuff but now they just let me get on with it." Jess (FG5). Adolescents express hope for their future occupational self, including articulating personal aims, dreams and aspirations, with particular importance placed on acquiring a good job and connecting with friends and family.

\section{Domain: Interacting with the situational context and the emerging occupational} self.

The most evident situational context transition for the adolescent is increased freedom over their own world, providing an opportunity to experience the consequences of both good and bad choices. 'So at home I am free to do whatever because after my GCSE's they saw how 
much my chilled out nature still is good enough... I've got freedom now. I wouldn't say I had that last year." Barry (FG3). There is evidence that adolescents experiment with occupational choice, such as trying to do homework in different locations, or managing relationships with others that influence where an occupation is done and when.

The participants' desire to take increased control over their environment fluctuates; their desire to master domestic chores within the home is low, but the desire to travel independently of their parents is higher, to the extent that some adolescents in rural locations stated they took a part time job to pay for driving lessons in order to gain more travel freedom. This suggests that adolescents are weighing up their desire to be more independent against the other environmental factors. In contrast, some adolescents in an urban location had to negotiate a different type of restriction, as illustrated by Liz and Komal's discussion in FG2 about the restrictions on their desire to travel:

Liz: I want to travel more than just being in school....I want to explore...Komal: like travel and stuff which we can't...we have to be home before $5 . .$. we have strict parents and they are scared that something might happen to us...I feel they don't trust us but they do...they don't trust the other people.

The transcripts indicate that the support an individual receives from others influences the occupational transition and whether an occupation is adopted or rejected as an occupational preference. "I think it [parental support] can be useful sometimes, they are right a lot of the time, but sometimes I think they should let me do it myself and learn from my mistakes." Tom (FG5). Adult support decreases as the adolescent develops skills competence. At the same time the adolescent becomes increasingly selective about what support and opinions 
they accept, as illustrated by one participant ( $F G 4$ ) (unable to identify which boy) talking about the influence of others “quite a bit by close friends but then sometimes you don't care what people think about it (occupation). If you want to do it then that's it." Another participant stated "the more people that know then the more people have an opinion on what you do and then they can be either more negative than positive and then you can get a bit put off by it" Mathew (FG4). Increased competence is accompanied by increased responsibilities to juggle, such as expectations to do chores or cook their supper while parents are at work. “...my mom washes the clothes and things before work. She tells me to fold them and put them back in the cupboard and stuff like that" Liz (FG2).

\section{Domain: Exploring skills and the occupational repertoire in relation to the emerging occupational self}

Adolescents experience a skills gap which requires them to juggle and weigh up previous experiences and their own personal occupational choices, in order to choose how best to address their skills gap. Their previous experience of engaging in an occupation, including both competence and achievement, appears to shape their expectations of success, the likelihood that they will persevere in the face of obstacles or difficulty, the methods chosen to develop a skill and indeed the likelihood that they will develop the skill successfully. For example, Natalie (FG1) talked about her experiences of auditions 'No matter how hard it gets.....you have to like put...yourself in the mind-set that you can do it." The experience of competence or achievement influences if an occupation is accepted or rejected. Therefore poor competence experiences impact skill development, and, in some cases, may adversely impact their ability to meet their need.

There is evidence in the narratives that some previously valued occupations are being discontinued while others are being prioritized and perfected, alternatively new occupations 
are developed. Many of the new skills involve new roles in new contexts, such as getting a job or learning to drive. There is an awareness of required standards of competence and an aspiration towards it, with varying levels of success. Each experience provides an opportunity to evaluate and improve competence.

\section{Domain: Considering time factors in relation to the emerging self}

Adolescents are increasingly structuring their own time, which involves practicing and developing the required skills. They experience increased self-responsibility and time conflicts, which can impact on how they feel: "you can be doing something more productive, but you're wasting your time" Rob (FG4). Learning to manage time factors can result in procrastination and unwise use of time, especially when not under the pressure of exams or deadlines. 'I can choose not to use my phone but I still do, but it could be also like my mom's given me some other work to do so I'm occupied there so I can't do my own work." Liz (FG2).

\section{Appraising and evaluating the four domains and the emerging self}

The transition from an externally structured world to their own internally constructed world that occurs across all four domains, is complex to negotiate, especially when adolescents are required to take increased responsibility for their choices concurrently with developing the ability to make those choices. Mathew's (FG4) describes, "sometimes it's hard to find the balance between, cause you only need to do school work, but if you have a football match or something that you're committed to you can't just leave so you work after." Although they are aware of their own preferences and reasons for engaging in occupation, they are still in a process of developing their occupational self and as such are still influenced by how chosen occupations may be judged by their parents, their peers and the educational system of which 
they are part. "I think it's like at least when...I'm trying to decide what to do. I think what will be the best story" Mathew (FG4).

The participants are aware of their growing freedom and autonomy to choose, even to choose socially unacceptable behaviours, and to experiment with new occupations. Through each experience of weighing up and juggling the four domains, making a choice and experiencing the consequences of that choice, they develop the four domains influencing future choices and shaping their future occupational self.

\section{Discussion}

The proposed theory describes a process by which adolescents make choices about what occupations they engage in, what affects how they choose to juggle activities and how those choices shape occupational development. Aligning with existing developmental literature, this theory provides unique insights, from an occupational science perspective, on the conscious process by which adolescents make, develop and adapt choices about the occupations they do. This includes considering contextual and individual opportunities and constraints. The theory provides new insights that complement wider developmental literature, existing occupation-based theories and contributes new conceptual renderings. In keeping with the constructivist grounded theory approach, the findings are discussed in relation to the literature.

Firstly, the proposed theory suggests a mechanism for occupational development, which meets three validity criteria for life course developmental theory by Baltes [38]: selection in 
favour of specific desired characteristics or functions, optimization towards improved functioning and efficacy, and finally compensation results from the fact that to develop new characteristics or abilities something else is lost. For example, adolescents articulated the value and prioritization of future employment or career aspirations over other occupations, exemplifying occupational selection. While 'optimisation' is articulated in aspirations and strivings towards adult task competencies, 'compensation' is evident in the loss of previously valued occupations as a direct result of developing newly prioritized characteristics or abilities, such as those associated with gaining their future adult self. Previously valued occupations may be stopped in preference for occupations associated with higher value or priority to their future occupational self.

Secondly, parallels can be drawn between this study's findings and developmental theories such as stage theories, decision-making theories, and theories of moral development among others. For example, Erikson's ideas on resolving identity conflict versus role confusion stage in late adolescence $[39,40]$ are evident but are viewed from an occupational perspective. Specifically, adolescents make a choice based on weighing up and juggling the four domains and appraising their experiences in relation to the four domains through which the occupational self is developed. Internal and external conflict are potential consequences of this process. For example: parental imposed restrictions on the situational context may prevent engaging in a valued or prioritized occupation, potentially creating a source of conflict, necessitating a response that draws on their developing skills and occupational repertoire. The resulting outcome may potentially affect an individual's sense of mental health and wellbeing. Other theories such as Harvinghurst's ideas about personal ideology development in 12 to 18 years $[40,41]$ also resonate, as do theories relating to the development of autonomy, independence, individualism and ego development [31]. These 
theories can be linked to the development of responsibility which is a prominent feature in the proposed theory.

Thirdly, a commonality with established existing occupation based theories, such as the Model of Human Occupation [4], is that occupational choice arises from experiencing need within the context of the environment and then leads to a response involving engagement in an occupation. Similarly, ideas evidenced within the widely accepted Self-Determination Theory $[43,44]$ such as autonomy, competence, and relatedness, are evident in the proposed theory. Study participants articulate the transition toward increased autonomy, developing competence, and discuss their connectedness with others in relation to making occupational choices. The proposed theory's credibility is thus strengthened by commonalities with established theories, but also contributes new insights. Figure 5 summarizes the specifics of the proposed theory, further discussed below.

\section{Insert Figure 5 here}

\section{The fit between existing skills, new occupational challenges and the impact on} wellbeing.

The first insight relates to the concept of 'the just right challenge'. It describes the balance between the level of challenge an occupation presents in relation to the skills and competencies an individual has to successfully complete it, which can impact both occupation completion and wellbeing [45]. It was first identified as an essential aspect of flow in Csikszentmihalyi's theory of optimal experience $[46,47]$. This concept has received little attention within the literature from an adolescent perspective, but was evident in the experiences reported in this study. Specifically, adolescent participants were focused on developing new competences and capabilities, drawing on past experience, which in turn impacted on feelings of competence, and skills development. Occupations deemed childish or 
too easy were avoided or discontinued in favour of challenges focusing on future aspirations. This suggests that late adolescents are actively engaged in a process of applying existing abilities to develop new ones. The 'fit 'between drawing on old abilities to develop new ones may influence the development of occupation competencies.

\section{An integrated view of interaction between the internal and external world}

The second insight relates to the common occupational conceptualization of a person's internal world, external environment and the interface between the two as described in models such as MOHO and CMOP-E. As compared to these models, which do emphasise the influence of the environment of occupations performed, the proposed theory suggests a more complex, multi-directional, integrated and ongoing interaction between the adolescents' developing internal world and their evolving external world, involving weighing up and juggling the four domains, and their resulting experience in the context of frequently overlooked temporal and developmental aspects. The process of choosing an occupation and its effect on the individual is perhaps more reflective of a complex systems approach, such as that described by Kantartzis [48] where change is viewed as a dynamic, ongoing, process that occurs both through the interaction within the system, and with the system and its envrionment. Potentially, this also addresses criticisms regarding the cross-cultural transferability of occupational theory models $[49,50]$. Especially in relation making occupational choice, such as the view point forward by Gallagher, Pettigrew, and Muldoon [51] who highlight that those in deprived areas with limited resources have little or no occupational choice. 


\section{The complexity of achieving occupational balance and the consequence of poor}

balance on wellbeing.

The third insight concerns how the proposed theory dynamically demonstrates the interrelated, and commonly used, theoretical concepts of occupational balance, patterns of daily occupations and their impact on health and wellbeing. It confirms the findings of a recent literature review that occupational balance is a subjective experience, and that patterns of daily occupation are a contexualised objective experience occurring within one's environment [9]. That review further suggests that occupational balance concerns one's ability and resource, congruence (of occupation) with values and meaning, and the harmonic mix of occupations, while patterns of daily occupation concerns the alteration, taxonomies and complexity of occupation from a time use perspective. These concepts are strongly evident in the proposed theory. For example, parallels can be drawn between abilities and resources and the skills and occupational répertoire domain, and between a 'harmonic mix' of activities and the effort of juggling and weighing up the different domains to best meet multiple, and often conflicting, needs.

The findings suggest that when occupational balance is not achieved, adolescent health and wellbeing is affected. Adolescents described the objective realities and complexities of taking responsibility for choosing their occupations, and the subjective impact of attempting to achieve occupational balance on their feeling of wellbeing. Examples include feelings of pressure or stress in relation to the need to complete schoolwork, experiencing time constraints, conflicting value and priorities, which results in healthy or unhealthy occupational choices. In some cases, this led to unhealthy occupational patterns that could be identified by the individual subjectively and objectively as unhealthy. These findings support 
the view that the balance and pattern of everyday occupations in which adolescents engage influence health.

\section{The challenge and complexity of attempting occupational balance while also developing}

The penultimate insight suggests that adolescent occupational choice is a complex and challenging process, where the adolescent is increasingly required to decide how best to identify and meet their own needs, and develop the skills required to meet the need within their own unique situational context. An example of this complexity is time management, where the adolescent juggles their immediately presenting needs against choices that meet longer term aspirations.

Another significant example is the cases where adolescents had migrated to the UK. Most described the challenge of adapting to a new environment, and the impact that had on the occupations they chose to do, and in some cases, this had resulted in the loss of valued occupations. They reported challenges weighing up and juggling conflicting situational contexts, as well as values and priorities domains, such as the challenge of juggling UK culture experienced at school, and that experienced in their home or the communities of which they were a part. The impact of this on wellbeing was less clear but it was evident that factors within the situational context were potentially an additional challenge for adolescents to manage. Adolescent responses to this potential stressor varied, but in some cases, it created an additional barrier to help seeking and was described as having a negative impact on their mental wellbeing. Indeed, in other recent research, the situational context has been identified as important in shaping occupational choices with potential impact on wellbeing [17, 52-53]. In particular, Galvaan's [17] work with marginalised youth in South Africa highlighted that 
the social environment together with the collective and contextual histories of an individual can shape not only the occupational opportunities but also which occupations are engaged in. This area would benefit from further exploration using grounded theory approaches.

\section{Experience shapes the emerging adolescent occupational self}

The final insight relates to the emerging occupational self. The proposed theory describes how, through experience, adolescents develop the ability to meet their needs, and how the experience of engaging in a chosen occupation shapes the development of a personalized version of the four domains, which in turn leads to development of the individual's future occupational self. Considered in relation to existing models and occupational theory, such as the Model of Human Occupation [4], the proposed theory provides a more in-depth and contextualized view of the potential interactive mechanisms that make up occupational choice and their influence on adolescent development and wellbeing.

Most significantly, the proposed theory differs from traditional occupation-based theories in that its foci is on the typical adolescent's process of making an occupational choice in the context of their environment and how the environment also shapes that process. It focuses on the domains that influence adolescent's choice and how they interact through the process. This is in contrast to existing models in which choices appear to be based on internal intentions

\section{Practical applications in OT}

Occupational therapists working with adolescents have previously drawn from psychological theories related to adolescent behaviour and stage theories of development to inform their practice. This knowledge has been used in conjunction with existing occupation based theories which do not fully illuminate the adolescent life stage The proposed theory, 
represents the complexity of making an occupational choice, and illuminates how that choice informs the individual's development from an occupational perspective. The theory offers a holistic and dynamic complex view of developmental transition, in relation to occupational choice, which aligns well with and supplements both existing developmental and occupation based theories.

The proposed theory also provides a rationale for how typical occupational skill development and occupational identity development occurs in late adolescence, and potentially how it could be impacted by illness or biographical disruption. Such knowledge can be used to inform both preventative and targeted interventions for those aged 16 to 17 years at risk of disrupted occupational development. The strong alignment between the emergent theory and ideas related to occupational balance suggests knowledge of weighing up and juggling the four domains can be used to enable adolescents to achieve a more healthy occupational balance, potentially leading to improved mental health and wellbeing. Consequently, the proposed theory may be of interest to occupational therapists and others seeking to promote healthy time use behaviours, occupational balance or develop occupation based interventions for late adolescent populations.

\section{Methodological considerations and limitations}

This study was designed as a pragmatic, small-scale feasibility study. Therefore, generalization is applied cautiously and further studies would be of value. All methodologies have limitations and it is possible that the self-selecting nature of the sample and choice of focus group over individual interviews may also have influenced the nature of responses given, both in terms of the information shared during the group and who chose to take part in the study. Theoretical sampling was also limited because of the school based sampling 
method, although saturation appeared to have been achieved which adds credibility. However, the study has significant strengths including the fact that study participants came from two significantly demographically different schools, providing culture related insights and furthermore the 'fit' with other substantiated theories boosts confidence in the proposed theory's representation of the late adolescent population. The use of a constructivist grounded theory is also a further strength of this study providing a structured approach to theory generation grounded in the data, which attempts to place prior knowledge to one side to avoid bias, whilst also acknowledging the co-created nature of the research process. It is acknowledged that the fact that the primary researcher's clinical experience as an occupational therapist and her familiarity with practice models influenced the questions asked in the focus groups. Similarly, familiarity with the core concepts of occupational science may have influenced the initial analysis process and construction of the emerging theory to some extent, accounting for this alignment with the meta-theory of occupational science.

The study specifically addressed how late adolescents make occupational choices. The relationship between health and the process of choosing an occupation emerged to towards the end of the time allocated for the focus group. This prevented detailed discussion about the impact of occupation on health. Specifically, participants tended to normalize subjects relating to health and wellbeing such as stress, conflicts, and tiredness within the context of everyday life. The fact that the participant population was generally a healthy one means that the findings of this study may not be representative of those with more severe health difficulties such as mental health problems. 
Further research is therefore required to examine the generalizability of the proposed theory both to the broader adolescent population and to specific groups such as those with mental health illness. The exploration of the relationship been adolescent choices and the experience of health and wellbeing would also be a valuable area of further injury.

There is also potential for future research to develop the emergent themes, including exploring how the theory may vary across age groups, gender or cultural factors.

\section{Conclusions}

This study has specifically explored the experiences of choosing occupations as reported by 16-17 years old, sampled from a school cohort of typical adolescents. In doing so, the emergent theory reflects both developmental and occupational perspectives from the subjective standpoint of the adolescent. The study suggests that the process of choosing a role, habit or project in which to engage, known as occupational choice, is a complex process shaped by the dynamic interaction of an individual's internal and external worlds. Direct occupational experiences appear to shape personal development and formation of occupational identity in adolescence. The study also highlights the challenge encountered by adolescents in making choices to meet their own needs at the same time as learning and developing the skills required to recognise and meet those same needs. This necessitates a regular negotiation between the choice to draw on existing skills, or engage with the challenge of developing new skills. The proposed theory aligns well with existing theory and contributes new insights into how young people make choices, how that shapes what they do, in the present and the future. It also provides insight into mechanisms, which may influence health and wellbeing during this critical period of development, and is consequently of use to those interested in this population and developing occupation-based interventions. 


\section{Acknowledgement}

With thanks to the Elizabeth Casson Trust for funding the project.

Professor Helen Dawes is supported by the Elizabeth Casson Trust and the NIHR Oxford Health Biomedical Research Centre.

The views expressed are those of the authors and not necessarily those of the NHS, the NIHR Department of Health or the Elizabeth Casson Trust.

\section{Disclosure statement}

The authors declare they have no conflicts of interest.

\section{Data availability statement}

Due to recent changes in legislation and the introduction of the GDPR the data is not open access. 


\section{References}

1. Lassi ZS, Salam R, Das JK, et al. An unfinished agenda on adolescent health: opportunities for intervetnions. Semin Perinatol 2015;353-60.

2. Viner RM, Ozer EM, Denny S, et al. Adolescence and the social determinants of health. Lancet. 2012;1641-1652.

3. Patton G, Viner R. Pubertal transitions in health. Lancet. 2007;1130-1139.

4. Taylor RR. Kielhofner's Model of Human Occupation: theory and application. 5th ed. Baltimore: Baltimore: Lippincott Williams \& Wilkins; 2017.

5. Coleman J, The nature of adolescence. London, Routledge. 2010.

6. Arnett JJ. 'Oh, grow up! generational grumbling and the new life stage of emerging adulthood-commentary on Trzesniewski \& Donnellan'. Perspect Psychol Sci. 2010;5:89-92.

7. Sawyer SM, Afifi RA, Bearinger LH, et al. Adolescence: a foundation for future health. Lancet. 2012;379:1630-1640.

8. Wilcock AA. Reflections on doing, being and becoming. Can J Occup Ther. 1999;65:248-256.

9. Eklund $M$, Kristina $O$, Argentzell $E$, et al. The linkage between patterns of daily occupations and occupational balance: applications within occupational science and occupational therapy practice. Scand J Occup Ther. 2017;41-56.

10. Eklund $M$, Leufstadius $C$. Relationships between occupational factors and health and wellbeing in individuals with persistent mental illness living in the community. Can J Occup Ther. 2007:04:303-13.

11. Persson $D$, Erlandsson L-K, Eklund $M$, et al. Value dimensions, meaning, and complexity in human occupation - a tentative structure for analysis. Scand J Occup Ther. 2009;8:7-18.

12. Eklund M, Erlandsson LK, Persson D. Occupational value among individuals with long-term mental illness. Can J Occup Ther. 2003;70:276-84.

13. Firth J, Ward P, Stubbs B. Editorial: lifestyle psychiatry. Front Psychiatry. 2019;10:597.

14. Medical Research Council. Developing and evaluating complex interventions: new guidance. Medical Research Council. 2006.

15. Hunt E, McKay E. What can be learned from adolescent time diary research. J Adolesc Health. 2015;56:256-266.

16. Widmark E, Fristedt S. Occupation according to adolescents: daily occupations categorized based on adolescents' experiences. J Occup Sci. 2018;26:470-483.

17. Galvaan R. The contextually situated nature of occupational choice: marginalised young adolescents' experiences in South Africa'. J Occup Sci. 2015;22:39-53.

18. Gallagher M, Pettigrew J, Muldoon O. Occupational choice of youth in a disadvantaged community. Br J Occup Ther. 2015;78:622-629.

19. World Health Organisation. Adolescent mental health [internet]. 2018 [Updated 2019 Oct]. Available from: https://www.who.int/news-room/fact-sheets/detail/adolescent-mentalhealth.

20. Kessler R, Amminger P, Aguilar-Gaxiola S, et al. Age of onset of mental disorders: a review of recent literature. Curr Opin Psychiatry. 2007;20:359-364. 
21. Patel V, Saxena S, Lund C, et al. The Lancet commission on global mental health and sustainable development. Lancet. 2018;392:1553-98.

22. Patel V, Flisher A J, Hetrick $S$, et al. Mental health of young people: a global public-health challenge. Lancet. 2007;369:1302-12.

23. Mei C, Fitzsimons J, Allen N, et al. 'Global research priorities for youth mental health '. Early Interv Psychiatry. 2020;14:3-13.

24. Hammarberg K, Kirkman M, de Lacey S. Qualitative research methods: when to use them and how to judge them. Human Reproduction. 2016;31:498-501.

25. Corbin J, Strauss A. Basics of qualitative research: techniques and procedures for developing grounded theory. 4th ed. London, SAGE. 2015.

26. Charmaz K, Belgrave L. Thinking about data with grounded theory. Qual Inq. 2018:1-11.

27. Birks M, Mills J. Grounded theory: a practical guide. London, SAGE. 2011.

28. Charmaz K. Special Invited Paper: continuities, contradictions, and critical inquiry in grounded theory. Int J Qual Methods. 2017;16:1-8.

29. Charmaz K. Constructing grounded theory: a practical guide through qualitative analysis. London, Sage. 2006.

30. Kamberelis G, Dimitriadis G. Focus groups: contingent articulations of pedagogy, polotics, and inquiry. In: Denzin N, Lincoln Y, editors. The SAGE Handbook of Qualitative Research. London, SAGE. 2011;545-562.

31. Kitzinger J. Qualitative research: introducing focus groups. BMJ. 1995;311:299-302.

32. Kamberelis G, Dimitriadis G. Focus groups: contingent articulations of pedagogy, polotics, and inquiry. In: Denzin, N. \& Lincoln, Y. (eds.) The SAGE Handbook of Qualitative Research. London, SAGE. 2011.

33. The World Medical Association. World medical association declaration of Helsinki. Ethical Principles for Medical Research Involving Human Subjects. Bull World Health Organ. 2011;373-374.

34. Ediger J, O'Day K. Occupation participation sort: adolescent and young adult. Innovative Practice Projects. 2011. Availble from:

https://pdfs.semanticscholar.org/f4c8/b5af4408d83adb35364cb7a605755b1503cf.pdf

35. Berg C, McCollum M, Cho E, et al. Development of the adolescent and young adult activity card sort. OTJR. 2015;35:221-231.

36. Hennink MM. Focus group discussion: understanding qualitative research. Oxford University Press. 2014.

37. Kitzinger J. The methodology of focus groups: the importance of interaction between research participants. Sociol Health IIIn. 1994;16:103-121.

38. Baltes PB. On the incomplete architecture of human ontogeny; Selection, optimization, and compensation as foundation of developmental theory. Am Psychol. 1997;52:366-380.

39. Sokol JT. Identity development throughout the lifetime: an examination of Eriksonian theory. Graduate Journal of Counseling Psychology. 2009:1:139-149.

40. Wright R, Sugarman L. Occupational therapy and life course development: a work book for professional practice. Singapore, Wiley-Blackwell. 2009. 
41. Havinghurst RJ. Developmental tasks and education. New York, David McKay. 1972.

42. Steinberg L, Cauffman E. Maturity of judgement in adolescence: psychosocial factors in adolescent decision making. Law Hum Behav. 1996;20:249-272.

43. Ryan RM, Decci EL. Self-determination theory and the facilitation of intrinsic motivation, social development, and well-being. Am Psychol. 2000;55:68-78.

44. Koole $S$, Schlinkert $C$, Maldei T, et al. Becoming who you are: an intergrative review of self determination theory and personailty systems interactions theory. J Pers. 2018;87:15-36.

45. Yerxa EJ. Occupational Science: A renaissance of service to humankind through knowledge. Occup Ther Int. 2000;7:87-98.

46. Rebeiro KL, Polgar J, M. Enabling occupational performance: optimal experiences in therapy Can J Occup Ther. 1999;66:14-22.

47. Csíkszentmihályi M. Flow: The psychology of optimal experience. New York, Harper and Row. 1990.

48. ntartzis S. The Dr Elizabeth Casson memorial mecture 2019: shifting our focus: fostering the potential of occupation and occupational therapy in a complex world. Br J Occup Ther. 2019;82:553-566.

49. Wong SR, Fisher G. Compairing and using occuaptional focused models. Occup Ther Health Care. 2015;29:297-315.

50. Iwama MK, Thomson NA, Macdonald RM. The Kawa model: The power of culturally responsive occupational therapy. Disabil Rehabil. 2009;31:1125-1135.

51. Gallagher M, Pettigrew J, Muldoon O. Occupational choice of youth in a disadvantaged community. Br J Occup Ther. 2015;78:622-629.

52. Nagle S, Cook JV, Polatajko HJ. I'm doing as much as I can: occupational choices of persons with a severe and persistent mental illness'. J Occup Sci. 2002;9:72-81.

53. Bonder BR. Culture and occupation: a comparison of weaving in two traditions. Can J Occup Ther. 2001;68:310-319. 